\title{
Éditorial
}

\section{Le DPC : Quelles évolutions ? Quels acquis?}

a formation continue est une obligation déontologique. Le Développement Professionnel Continu (DPC) est Leffectif depuis 2013 et obligatoire pour tous les professionnels de santé. ll a pour objectif principal l'amélioration de la qualité et de la sécurité des soins pour une meilleure prise en charge des patients.

Le but du DPC est d'actualiser ses connaissances par un parcours personnalisé et contrôlé.

Malgré les contraintes administratives, le DPC est une approche scientifique novatrice et bénéfique pour le praticien comme pour le patient. Le non-suivi de ce plan personnalisé peut constituer un cas d'insuffisance professionnelle.

\section{Que nous apporte le DPC ?}

La différence avec la formation continue habituelle réside dans l'évaluation : c'est un changement dans la façon d'appréhender sa formation continue :

- Évaluation de ses connaissances en amont et repérage de ses besoins ;

- Recherche de la Formation DPC (orientations nationales);

- Évaluation de ses acquis pour améliorer sa pratique professionnelle et la sécurité des patients.

\section{Quelle évolution pour la formation continue?}

Suite à la réforme du DPC prévue dans la loi Touraine, la réglementation vient de subir des modifications :

- En mai 2015, l'Ordre et la profession ont créé le Conseil National Professionnel (CNP), dont l'objectif est de parvenir à une formation continue et une évaluation des pratiques axées autour des vrais besoins des chirurgiens-dentistes : il sera l'interlocuteur de la profession avec les pouvoirs publics.

- Jeudi 17 décembre 2015, la loi de Modernisation de notre système de Santé a été adoptée par l'Assemblée nationale :

- L'obligation de suivre un programme de DPC passera de un DPC par an à un DPC tous les 3 ans dès la parution des décrets d'application. Chaque praticien doit désormais se connecter sur son compte personnel en allant sur www.mondpc.fr depuis le moteur de recherche disponible sur www.ogdpc.fr; 
- Création des orientations nationales du développement professionnel continu des professionnels de santé pour les années 2016 à 2018. Les programmes de DPC devront entrer dans ces orientations (selon l'arrêté du 8 décembre 2015);

- Création d'un « portfolio » (en cours de réalisation) car la formation continue ne se résume pas au seul DPC. Chaque praticien conserve le droit (et même le devoir) de faire librement d'autres formations et devra inscrire dans ce document son parcours de formation continue tout au long de sa carrière professionnelle. On peut compléter ses connaissances par différents moyens : cours, congrès, séminaires, lectures, groupes de travail, e-learning, etc.

Il est donc fondamental pour les praticiens de tenir à jour toutes les formations suivies. Ceci pourra constituer une preuve vis-à-vis des tribunaux en cas de litige avec un patient ou d'aléa thérapeutique.

\section{Une autre piste pour la qualité des soins et l'actualisation des connaissances : les recommandations de bonne pratique}

Les recommandations professionnelles faites par la Haute Autorité en Santé (HAS) en orthodontie sont anciennes et leur actualisation n'est pas prévue dans l'immédiat. La profession, et en particulier les professionnels concernés par l'orthodontie, ont décidé de mettre en œuvre des recommandations de bonnes pratiques par consensus d'experts.

Deux recommandations sont parues en 2015 :

- Prise en charge d'une canine incluse ;

- Maîtrise du risque infectieux dans le cadre de la réalisation des actes d'orthodontie.

C'est la profession toute entière qui s'implique dans la qualité des soins.

Claude Bourdillat-Mikol

Présidente de la commission Formation Continue de la FFO

\section{Extrait de l'arrêté du 8 décembre 2015 fixant la liste des orientations nationales pour les professionnels de santé concernant l'orthodontie pour les années 2016 à 2018}

Chirurgien-dentiste spécialisé en orthopédie dento-faciale :

Orientation $n^{\circ} 1$ : innovation en orthopédie dento-faciale

Orientation $n^{\circ} 2$ : nouvelles recommandations en orthopédie dento-faciale

Orientation $n^{\circ} 3$ : gestion des risques associés aux actes et aux modalités de prise en orthopédie dento-faciale

Les opinions émises n'engagent que leurs auteurs. 